\title{
Prognostic significance of leucine-rich-repeat-containing G-protein-coupled receptor 5, an intestinal stem cell marker, in gastric carcinomas
}

\author{
Bo Gun Jang ${ }^{1}$ Byung Lan Lee ${ }^{3}$ Woo Ho Kim $^{2}$
}

Received: 11 December 2014/Accepted: 7 September 2015/Published online: 19 September 2015

(c) The International Gastric Cancer Association and The Japanese Gastric Cancer Association 2015

\begin{abstract}
Background Cells expressing LGR5, an intestinal stem cell marker, have been suggested as cancer stem cells in human colon cancers. Previously, we discovered that LGR5-expressing cells exist in the gastric antrum and remarkably increase in number in intestinal metaplasia. In addition, most gastric adenomas contain abundant LGR5expressing cells coexpressing intestinal stem cell signatures. However, LGR5 expression in gastric cancers (GCs) and its prognostic significance remain unknown.

Methods We examined the LGR5 expression in GC tissues by real time-PCR and RNA in situ hybridization, and analyzed its clinicopathological relevance and prognostic value. The effects of LGR5 on cancer cell proliferation and migration were assessed with an in vitro transfection technique.

Results LGR5 expression was significantly lower in GCs than in matched nontumorous gastric mucosa. RNA in situ hybridization on tissue microarrays showed that $7 \%$ of GCs were positive for LGR5. LGR5 positivity was associated with old age, well to moderate differentiation, and nuclear $\beta$-catenin positivity. Although LGR5 did not show any prognostic significance for all GC cases, it was
\end{abstract}

Woo Ho Kim

woohokim@snu.ac.kr

1 Department of Pathology, Jeju National University Hospital, Jeju, Korea

2 Department of Pathology, Seoul National University College of Medicine, 28 Yeongeon-dong, Jongno-gu, Seoul 110-799, Korea

3 Department of Anatomy, Seoul National University College of Medicine, 28 Yeongeon-dong, Jongno-gu, Seoul 110-799, Korea associated with poor survival in GCs with nuclear $\beta$-catenin expression. LGR5 expression was induced by transfection in GC cell lines with abnormal Wnt activation, which, however, showed no influence on the growth and migration of GC cells.

Conclusion A small portion of GCs expressed LGR5. Although LGR5 was associated with poor survival in GCs with nuclear $\beta$-catenin, LGR5 expression in GC cells had no effects on the growth and migration, requiring a further study exploring a biological role of LGR5 in GCs.

Keywords LGR5 - Gastric cancer - In situ hybridization * Prognosis $\cdot$ Stem cells

\section{Introduction}

Gastric cancer (GC) is the fifth commonest malignancy in the world and the third leading cause of cancer death worldwide [1]. Despite remarkable progress in the diagnostic and therapeutic tools, patients with advanced GCs still have poor clinical outcomes. The molecular characterization of GCs has led to the identification of several promising targets, including vascular endothelial growth factor receptor 2, c-MET, fibroblast growth factor receptor 1, fibroblast growth factor receptor 2, human epidermal growth factor receptor 2 (HER2), and human epidermal growth factor receptor 3 [2]. Indeed, the anti-HER2 antibody trastuzumab in patients with GC overexpressing HER2 improved overall survival compared with standard chemotherapy [3]. However, the response rates remain in the $25-40 \%$ range across trials, and novel molecularly directed approaches are required [2].

Rapidly mounting evidence suggests that cancer stem cells not only maintain tumor growth but also result in 
relapse after therapy, suggesting they could be a candidate for new targeted therapy [4]. Leucine-rich-repeat-containing G-protein-coupled receptor 5 (LGR5) has been demonstrated as a global stem cell marker in multiple organs, including the small intestine, colon, stomach, hair follicle, kidney, ovary, liver, and mammary gland [5-11]. In these organs, LGR5 not only marks Wnt-driven stem cells that drive constitutive tissue self-renewal, but also defines a class of stem cells induced by tissue damage [11]. LGR5-expressing cells have been shown as the cells of origin of intestinal and gastric adenomas [6, 12]. Moreover, LGR5 has been suggested as a selective marker for human colorectal cancer stem cells [13].

Subsequent studies investigated the clinical and functional implications of LGR5 in a variety of human malignant tumors-for example, colorectal cancers [14-16], GCs [17, 18], and hepatocellular carcinomas [19]. Even in lung adenocarcinomas [20] and gliomas [21], LGR5 expression and its clinicopathological significance were examined although LGR5-expressing stem cells normally do not exist in the lung and brain. In colorectal cancers, the prognostic value of LGR5 was extensively studied and found to be statistically related to the reduced overall survival [22]. However, for GCs, only a limited number of studies have investigated LGR5 expression and its relevance in predicting clinical outcomes [17, 23, 24]. Previously, we specifically identified LGR5-expressing cells in normal gastric mucosa and intestinal metaplastic lesions as well as gastric adenomas by RNA in situ hybridization (ISH) [25]. In this study, we investigated the expression of LGR5 messenger RNA (mRNA) in a large number of GC patients and analyzed its significance in the prognosis of GC patients.

\section{Patients and methods}

\section{Patients}

Formalin-fixed and paraffin-embedded (FFPE) GCs were collected from 840 patients who underwent curative gastrectomy at Seoul National University Hospital, Seoul, Korea, from 2004 to 2005. We collected clinicopathology data, including patient age and sex, histological type, evidence of lymphovascular invasion, and TNM pathological stages, by reviewing the medical records and pathology records according to the seventh edition of the American Joint Committee on Cancer's cancer staging manual. Also, paired, freshly frozen GC tissues and matched noncancerous gastric tissues were available from $35 \mathrm{GC}$ patients from 2001 to 2005. A normal small intestine specimen was obtained from a patient with gastrointestinal stromal tumor of the small intestine who underwent curative segmental resection. This study was approved by the Institutional Review Board of Seoul National University Hospital (reference H-1209-037-424).

\section{Gastric carcinoma cell lines}

Fifteen human gastric carcinoma cell lines (SNU-1, SNU5, SNU-16, SNU-216, SNU-484, SNU-601, SNU-620, SNU-638, SNU-668, SNU-719, MKN-1, MKN-28, MKN45, MKN-74, and AGS) were obtained from the Korean Cell Line Bank (Seoul, Korea). Cell lines were cultured in RPMI 1640 medium containing $10 \%$ fetal bovine serum and antibiotics (penicillin $\mathrm{G}$ and streptomycin) in a humidified incubator containing $5 \% \mathrm{CO}_{2}$. The cell lines were subject to immunocytochemistry for $\beta$-catenin after formalin fixation for $1 \mathrm{~h}$.

\section{Tissue microarray construction}

Fourteen tissue microarrays containing $840 \mathrm{GCs}$ from gastrectomy specimens were generated. In brief, core tissue biopsies ( $2 \mathrm{~mm}$ in diameter) were obtained from individual FFPE gastric tumors (donor blocks) and arranged in a new recipient paraffin block (tissue array block) with a trephine apparatus (SuperBioChips Laboratories, Seoul, Korea).

\section{Immunohistochemistry}

We performed immunohistochemistry on $4-\mu \mathrm{m}$ tissue microarray sections using a BOND-MAX automated immunostainer and a Bond Polymer Refine Detection kit (Leica Microsystems, Wetzlar, Germany) according to the manufacturer's instructions. The primary antibody used was anti- $\beta$-catenin (Novocastra Laboratories, Newcastle, $\mathrm{UK} ; 17 \mathrm{C} 2 ; 1: 800$ ), and $\beta$-catenin staining was considered positive when more than $10 \%$ of the tumor cell nuclei were strongly stained for $\beta$-catenin.

\section{RNA ISH and interpretation}

ISH for LGR5 was performed with an RNAscope FFPE assay kit (Advanced Cell Diagnostics, Hayward, CA, USA) as described previously [25]. In brief, 4- $\mu \mathrm{m}$ FFPE tissue sections were pretreated with heat and protease digestion followed by hybridization with LGR5 probe. Then, a horseradish peroxidase based signal amplification system was hybridized to the LGR5 probe before color development with 3,3'-diaminobenzidine tetrahydrochloride. The housekeeping gene ubiquitin $\mathrm{C}(U B C)$ and the bacterial gene $\operatorname{dap} B$ served as a positive and a negative control, respectively. Samples with $U B C$ easily visible under a $\times 10$ objective lens were considered to be adequate according to the manufacturer's recommendation, and finally 603 GCs 
were included for the evaluation. Two gastrointestinal pathologists (B.G.J and G.H.K.) independently interpreted the staining of LGR5. For the discordant cases, a final decision was made by consensus. LGR5 staining was graded on the basis of the percentage of tumor cells positive for LGR5 as follows: grade $0,0-5 \%$; grade 1 , 5-10 \%; grade 2, 10-25 \%; and grade 3, 25-100 \%. When more than $5 \%$ of tumor cells express LGR5 (more than grade 1), the sample is considered as positive for LGR5.

\section{RNA extraction and quantitative real-time PCR}

We prepared total RNA from the 35 paired freshly frozen gastric tumors and corresponding normal gastric tissue samples as well as $15 \mathrm{GC}$ cell lines using TRIzol (Invitrogen, Carlsbad, CA, USA). Total RNA $(1 \mu \mathrm{g})$ was reverse transcribed with oligo-dT primers and the GoScript reverse transcription system (Promega, Madison, WI, USA). PCRs were performed with Premix EX Taq (Takara bio, Shiga, Japan) according to the manufacturer's recommendations, and the cycling conditions were followed: initial denaturation for $30 \mathrm{~s}$ at $95{ }^{\circ} \mathrm{C}$, followed by $40-50$ cycles of $95{ }^{\circ} \mathrm{C}$ for $5 \mathrm{~s}$ and $60{ }^{\circ} \mathrm{C}$ for $34 \mathrm{~s}$ in a 7500 real-time PCR system (Applied Biosystems, Foster City, CA, USA). The data were analyzed by the 7500 real-time PCR system software, SDS (version 1.4; Applied Biosystems). The TaqMan gene expression assays used were as follows: Hs00173664_m1 (LGR5), Hs00362096_m1 (EPHB2), Hs00270888_s1 (ASCL2), Hs00197437_m1 (OLFM4), and Hs0275899_g1 $(G A P D H)$. GAPDH served as the endogenous control, and all experiments were performed in duplicate.

\section{Western blot analysis}

Cellular proteins were extracted from the GC cells in lysis buffer (iNtRON Biotechnology, Seongnam, Korea), and protein levels were determined by bicinchoninic acid protein assay kits (Pierce, Rockford, IL, USA). The rabbit anti-LGR5 (Abcam, Cambridge, UK; ab75850) and mouse anti- $\alpha$-actin (Sigma-Aldrich, St Louis, MO, USA) antibodies were used as primary antibodies. After overnight incubation at $4{ }^{\circ} \mathrm{C}$ and washing with tris(hydroxymethyl)aminomethane-buffered saline containing $0.1 \%$ Tween 20, blots were incubated for $1 \mathrm{~h}$ at room temperature with secondary antibodies, and then washed and visualized with enhanced chemiluminescence kits (Pierce).

\section{Transfection with LGR5}

Full-length complementary DNA encoding LGR5 (pEXLGR5) was purchased from GeneCopoeia (Rockville, MD, USA). Cells were seeded at $1 \times 10^{6}$ cells per well in a sixwell plate and transfected with $2.5 \mu \mathrm{g}$ of pEX-LGR5 or control vector (pEX-EGFP) by means of Lipofectamine 3000 transfection reagent (Invitrogen, Carlsbad, CA, USA) according to the manufacturer's instructions. One day after transfection, cells were subjected to reverse transcription PCR (RT-PCR), Western blot, and a proliferation assay.

\section{Cell proliferation assay}

Twenty-fours hours after transfection in a six-well plate, cells were harvested and seeded at $5 \times 10^{3}$ cells per well on 96-well plates and incubated at $37{ }^{\circ} \mathrm{C}$. To examine the effect of LGR5 on the chemosensitivity, 5-fluorouracil (Sigma-Aldrich) was added at a concentration of $100 \mu \mathrm{g} /$ $\mathrm{ml}$ and vehicle (dimethyl sulfoxide) was added to control cells. After addition of $10 \mu \mathrm{l}$ of Cell Counting Kit-8 reagent (Dojindo, Tokyo, Japan) to each well and incubation for $1 \mathrm{~h}$, the absorbance was measured at $450 \mathrm{~nm}$ with a spectrophotometer (Thermo Labsystems, Beverly, MA, USA).

\section{Wound healing assay}

AGS and SNU-484 cells were cultured in six-well plates until they were confluent after transfection with the control vector or LGR5. The monolayer was scratched with a pipette tip to create a wound and washed twice with culture medium to remove cell debris. Cellular migration was monitored and photographed at 0 and $48 \mathrm{~h}$.

\section{Statistical analysis}

Statistical analyses were performed with the statistical software program PASW 18.0 (IBM SPSS Statistics, Chicago, IL, USA) and Prism version 5.0 (GraphPad Software, San Diego, CA, USA). The correlations between LGR5 positivity and clinicopathological parameters were tested by Fisher's exact test or Pearson's chisquare test. Between-group comparisons of the real-time PCR data were performed by Student's $t$ test. Survival curves were estimated by the Kaplan-Meier method, and the log-rank test was used to compare groups. The results were considered significant when $P<0.05$.

\section{Results}

\section{LGR5 expression in human GC}

To compare the expression of LGR5 between human GCs and noncancerous tissues, real-time PCR was done on a series of 35 pairs of freshly frozen human GC samples and matched noncancerous gastric mucosa. When we evaluated individual cancers, LGR5 expression 
of GC was lower than that of noncancerous tissues in most patients ( $80 \%, 28$ of 35 cases) (Fig. 1a). Overall, GCs expressed a significantly lower level of LGR5 mRNA than adjacent noncancerous gastric tissues $(P<0.01)$ (Fig. 1b). We previously demonstrated that LGR5-expressing cells in gastric adenomas coexpress other intestinal stem cell (ISC) signatures such as ASCL2, EPHB2 and OLFM4, which we thought suggested a stem cell feature of LGR5-expressing cells. To see whether GCs retain this relationship of LGR5 with ISC markers, we compared the expression levels of the ISC markers between LGR5-low and LGR5-high groups, which were divided by the value of 0.1 (relative mRNA level of $L G R 5$ to GAPDH). Only EPHB2 expression was slightly higher in the LGR5-high group than in the LGR5-low group (Fig. 1d). No difference was observed in the expression of OLFM4 and ASCL2 between groups (Fig. 1c, e), indicating a reduced connection of LGR5 with ISC markers in GCs.

\section{Association of LGR5 expression with clinicopathological parameters in GC}

RNA ISH on a small intestine clearly demonstrates specific marking of LGR5-expressing stem cells at the crypt bases as seen previously (Fig. 2a-c) [5]. No nonspecific staining was observed in any stromal cells or intestinal epithelial cells above crypt bottoms. RNA ISH was performed on the tissue microarrays containing 840 GCs. Finally, 603 cases were included for the analysis. In total, 42 cases $(7 \%)$ of GCs were positive for LGR5 (Fig. 2d-f). The clinicopathological relevance of LGR5 expression is shown in Table 1. LGR5 positivity was significantly higher in older age (older than 65 years) $(P=0.002)$, and GCs with welldifferentiated or papillary histological type expressed LGR5 more frequently than did poorly differentiated or signet ring cell carcinoma $(P=0.026)$. Immunohistochemical analysis for $\beta$-catenin was also performed to investigate the relationship between LGR5 and Wnt

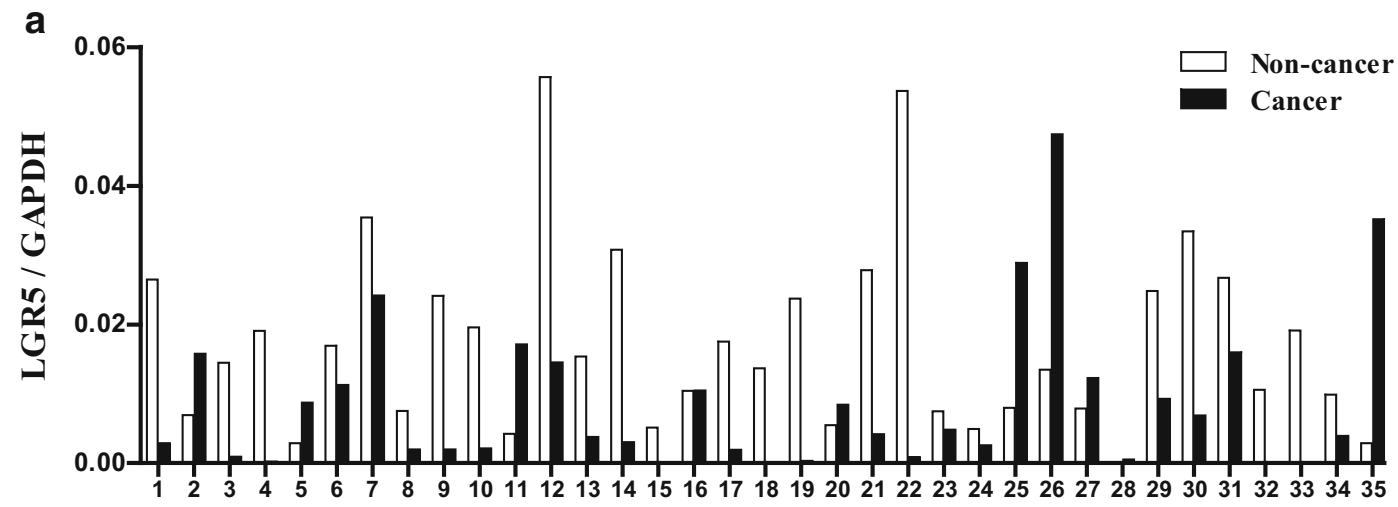

Patient
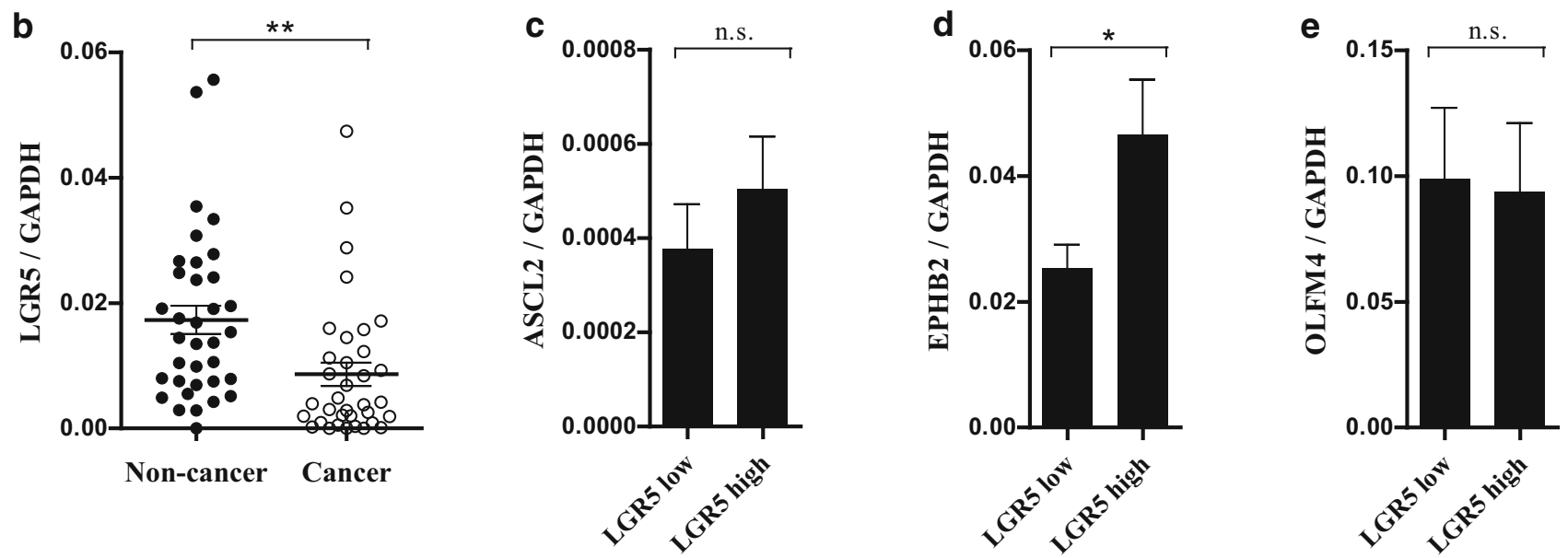

Fig. 1 LGR5 expression in gastric cancers and its correlation with other intestinal stem cell markers. a Real-time PCR analysis examined the expression of the intestinal stem cell markers LGR5, ASCL2, EPHB2, and OLFM4 with 35 pairs of gastric cancers and

corresponding noncancerous gastric mucosa. b-e The mean level of LGR5 in gastric cancers was significantly lower than in noncancerous gastric tissues, and was associated with EPHB2 expression, but not with OLFM4 and ASCL2 expression. n.s. not significant 


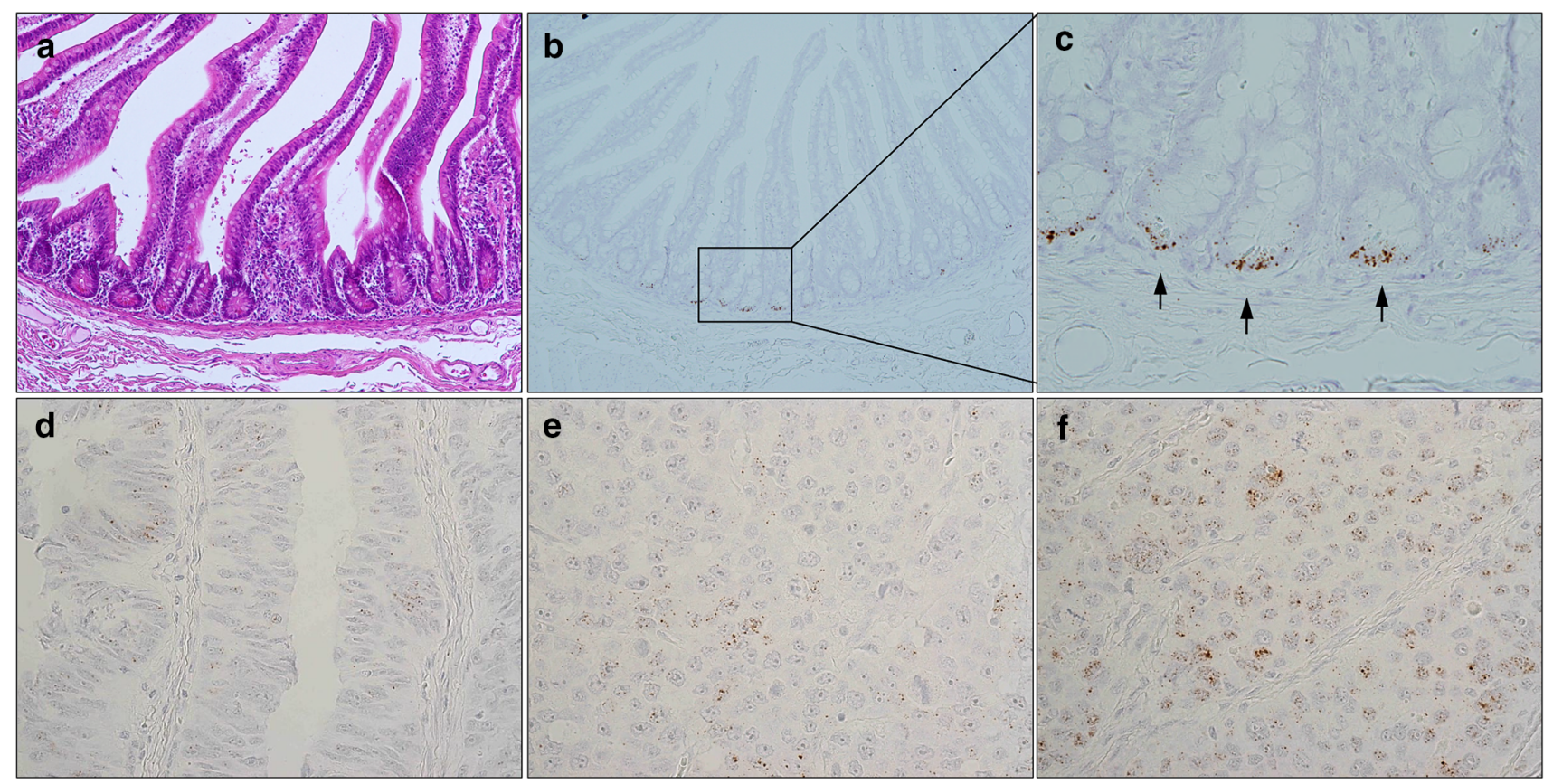

Fig. 2 RNA in situ hybridization (ISH) for LGR5 in gastric carcinomas. a-c RNA ISH on a specimen of human small intestine specifically identified LGR5 cells (arrows) at the bases of crypts. RNA ISH on tissue microarrays containing 840 gastric cancers was

pathway activation. LGR5 positivity showed a strong positive correlation with nuclear $\beta$-catenin expression (Fig. 3). However, LGR5 revealed no correlation with sex, location, lymphatic invasion, venous invasion, and TNM stage.

\section{Prognostic value of LGR5 in GC}

Next, we assessed the prognostic significance of LGR5 in GC patients using Kaplan-Meier analysis and found that LGR5 positivity had no correlation with overall survival ( $P=0.560$ ) (Fig. 4a). Recently, it has been shown that LGR5 functions as a ligand for R-spondins and potentiates the $\mathrm{Wnt} / \beta$-catenin pathway only when Wnt signaling is activated [26, 27], which led us to speculate that LGR5 expression could exert a synergistic effect on GCs harboring an abnormal Wnt pathway. Therefore, we tried to examine the prognostic effect of LGR5 in a subgroup of GC patients who had nuclear $\beta$ catenin expression $(n=34)$, indicative of enhanced Wnt signaling. Nuclear $\beta$-catenin positivity itself was not a prognostic marker in GCs (data not shown). Remarkably, LGR5-positive GC patients had worse clinical outcomes than LGR5-negative GC patients $(P=0.007)$ (Fig. 4b), suggesting LGR5 is a poor prognostic marker when the performed, and tumors were interpreted according to the percentage of LGR5 positive cells. $\mathbf{d}$ Grade 1 , $\mathbf{e}$ grade 2 , f grade 3 . a, $\mathbf{b} \times 100$; $\mathbf{c}-$ f $\times 400$

cases are restricted to the GCs with abnormally enhanced Wnt signaling.

\section{Effects of LGR5 expression on the growth and migration of GC cells}

As LGR5 positivity was shown to be a worse prognostic marker for GC patients with increased Wnt signaling, we figured that LGR5 could have a functional effect on cancer cell growth or migratory activity. LGR5 expression was examined in $15 \mathrm{GC}$ cell lines, and most of the GC cell lines, except AGS and SNU-620, showed extremely low levels of LGR5 mRNA (Fig. 5a). No strong correlation was observed between LGR5 and ISC markers in GC cells (Fig. 5b). The LGR5-high group exhibited only slightly higher levels of ASCL2 than the LGR5-low group (Fig. 5b). Four GC cell lines with nuclear $\beta$-catenin expression (AGS, SNU-719, MKN-28, and MKN-74) and one without nuclear $\beta$-catenin expression (SNU-484) were transfected with empty vector or LGR5-expressing vector (Fig. 5c). The expression of LGR5 was assessed by RTPCR analysis (Fig. 6a) and Western blot analysis (Fig. 6b) to demonstrate that LGR5 protein was expressed only in the AGS and SNU-484 cell lines, which were used for the functional experiments. The relative growth rate was 
Table 1 Association between LGR5 expression and the clinicopathological characteristics

\begin{tabular}{|c|c|c|c|c|}
\hline & \multirow[t]{2}{*}{ Total } & \multicolumn{2}{|l|}{ LGR5 } & \multirow[t]{2}{*}{$P$} \\
\hline & & Negative & Positive & \\
\hline Patients & $603(100 \%)$ & $561(93 \%)$ & $42(7 \%)$ & \\
\hline \multicolumn{5}{|l|}{ Age } \\
\hline$\geq 65$ years & $224(37 \%)$ & $199(89 \%)$ & $25(11 \%)$ & \multirow[t]{2}{*}{$0.002^{\mathrm{b}}$} \\
\hline$<65$ years & $379(63 \%)$ & $362(96 \%)$ & $17(4 \%)$ & \\
\hline \multicolumn{5}{|l|}{ Sex } \\
\hline Female & $173(29 \%)$ & $162(94 \%)$ & $11(6 \%)$ & \multirow[t]{2}{*}{$0.710^{\mathrm{b}}$} \\
\hline Male & $430(71 \%)$ & $399(93 \%)$ & $31(7 \%)$ & \\
\hline \multicolumn{5}{|c|}{ Histological differentiation } \\
\hline Well & $125(21 \%)$ & $108(86 \%)$ & $17(14 \%)$ & \multirow[t]{6}{*}{$0.026^{\mathrm{b}}$} \\
\hline Moderate & $240(40 \%)$ & $223(93 \%)$ & $17(7 \%)$ & \\
\hline Poor & $147(24 \%)$ & $143(97 \%)$ & $4(3 \%)$ & \\
\hline Signet ring cell & $62(10 \%)$ & $60(97 \%)$ & $2(3 \%)$ & \\
\hline Papillary & $15(3 \%)$ & $13(87 \%)$ & $2(13 \%)$ & \\
\hline Other types & $14(2 \%)$ & $14(100 \%)$ & $0(0 \%)$ & \\
\hline \multicolumn{5}{|l|}{ Location } \\
\hline Upper third & $90(15 \%)$ & $86(96 \%)$ & $4(4 \%)$ & \multirow[t]{4}{*}{$0.711^{\mathrm{c}}$} \\
\hline Middle third & $226(37 \%)$ & $208(92 \%)$ & $18(8 \%)$ & \\
\hline Lower third & $277(46 \%)$ & $258(93 \%)$ & $19(7 \%)$ & \\
\hline Whole & $10(2 \%)$ & $9(90 \%)$ & $1(10 \%)$ & \\
\hline \multicolumn{5}{|l|}{ Lymphatic invasion } \\
\hline Negative & $216(36 \%)$ & $205(95 \%)$ & $11(5 \%)$ & \multirow[t]{2}{*}{$0.186^{b}$} \\
\hline Positive & $387(64 \%)$ & $356(92 \%)$ & $31(8 \%)$ & \\
\hline \multicolumn{5}{|l|}{ Venous invasion } \\
\hline Negative & $499(83 \%)$ & $468(94 \%)$ & $31(6 \%)$ & \multirow[t]{2}{*}{$0.133^{b}$} \\
\hline Positive & $104(17 \%)$ & $93(89 \%)$ & $11(11 \%)$ & \\
\hline \multicolumn{5}{|l|}{ TNM stage ${ }^{\mathrm{a}}$} \\
\hline I & $167(27 \%)$ & $155(93 \%)$ & $12(7 \%)$ & \multirow[t]{4}{*}{$0.255^{\mathrm{c}}$} \\
\hline II & $163(27 \%)$ & $147(90 \%)$ & $16(10 \%)$ & \\
\hline III & $213(35 \%)$ & $198(93 \%)$ & $15(7 \%)$ & \\
\hline IV & $66(11 \%)$ & $61(92 \%)$ & $5(8 \%)$ & \\
\hline \multicolumn{5}{|l|}{$\beta$-Catenin } \\
\hline Nuclear stain & $34(6 \%)$ & $23(68 \%)$ & $11(32 \%)$ & \multirow[t]{2}{*}{$0.000^{\mathrm{b}}$} \\
\hline No nuclear stain & $569(94 \%)$ & $538(95 \%)$ & $31(5 \%)$ & \\
\hline
\end{tabular}

a American Joint Committee on Cancer's cancer staging manual, seventh edition

b Fisher's exact test

c Pearson's chi-square test measured 4 days after transfection. However, there was no difference in the proliferation between cancer cells transfected with LGR5 and those transfected with control vector (Fig. 6c). In addition, LGR5 overexpression had no influence on the antiproliferative effect induced by 5-fluorouracil (100 $\mu \mathrm{g} / \mathrm{ml})$ (Fig. 6d). The wound healing assay revealed that LGR5 expression had no impact on the migration activity of GC cells either (Fig. 6e). These findings suggest that in vitro LGR5 overexpression alone is not enough to change the characteristics of GCs. Probably, additional signaling factors along with the $\mathrm{Wnt} / \beta$-catenin pathway are necessary to alter the biological behavior of GCs.

\section{Discussion}

LGR5 is the most promising stem cell marker in the normal gastric and intestinal mucosa. Using monoclonal antibodies against human LGR5 developed in the laboratory, Kemper et al. [13] provided evidence that LGR5 is also a marker for human colorectal cancers. However, the possibility of its 


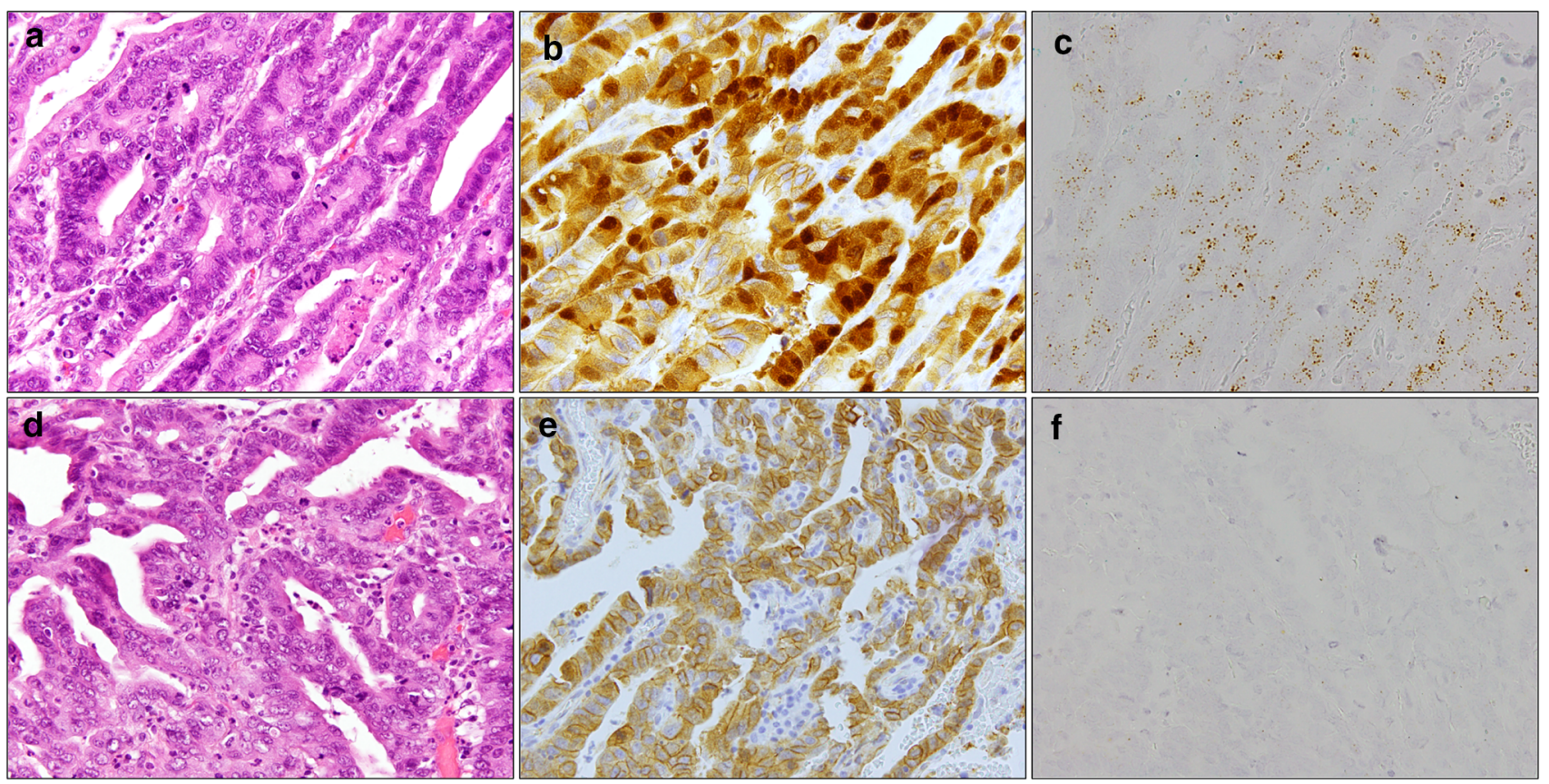

Fig. 3 Positive association of LGR5 expression with nuclear $\beta$ catenin positivity. A representative gastric cancer (a) with nuclear $\beta$ catenin expression (b) showed strong LGR5 expression (c), whereas

a

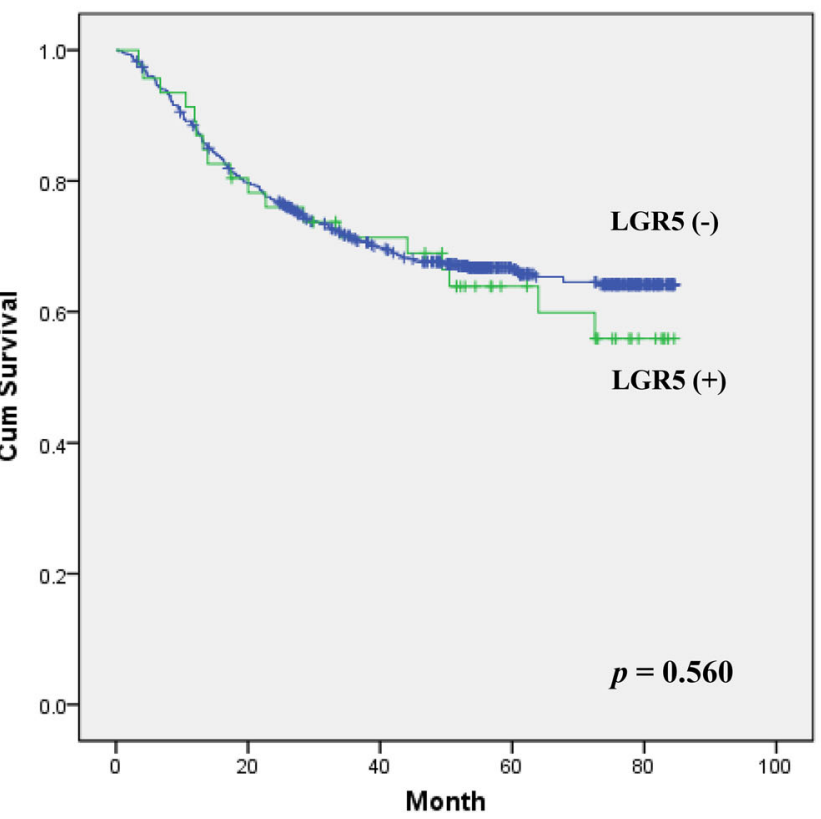

Fig. 4 Survival rate of gastric cancer patients with LGR5 expression. Kaplan-Meier analysis demonstrated that LGR5 positivity has no prognostic influence in gastric cancer patients $(n=603, P=0.560)$.

use as a stem cell marker in GCs has not been investigated yet even though LGR5 expression has been demonstrated through GC progression; normal gastric epithelium, other gastric cancer (d) with membranous $\beta$-catenin expression (e) exhibited no LGR5 expression (f). Magnification $\times 400$

b

Gastric cancers with

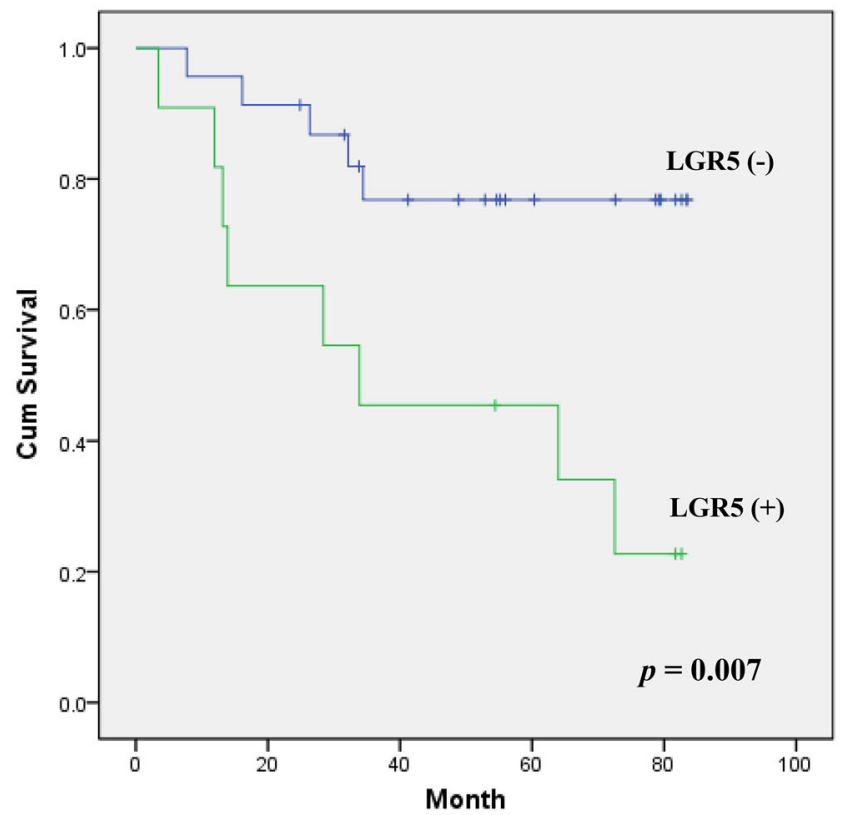

However, for the gastric cancers with nuclear $\beta$-catenin expression, LGR5 expression was associated with poor survival $(n=34$, $P=0.007)$. Cum cumulative

precancerous lesion, adenoma, and carcinoma [17, 25]. Because of the lack of commercially available antibodies that can recognize the extracellular domain and isolate 


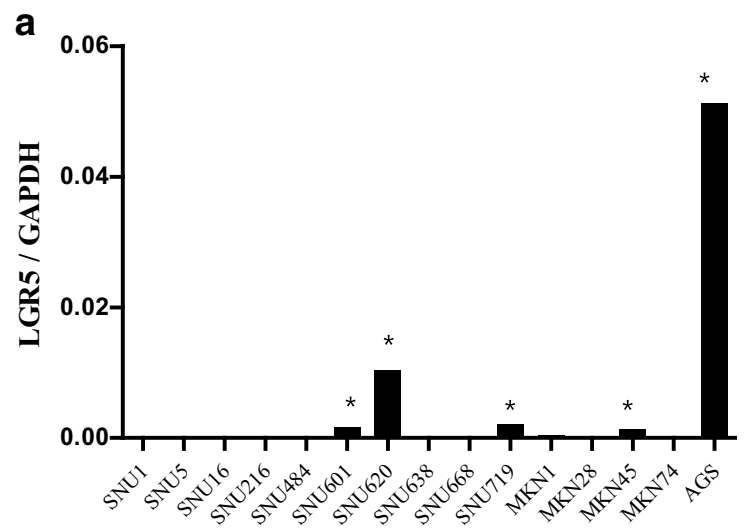

b
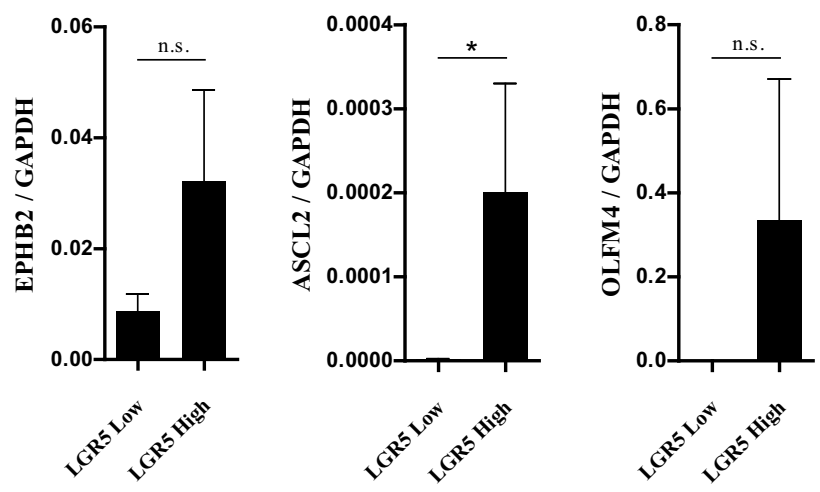

C

Nuclear $\beta$-catenin (+)
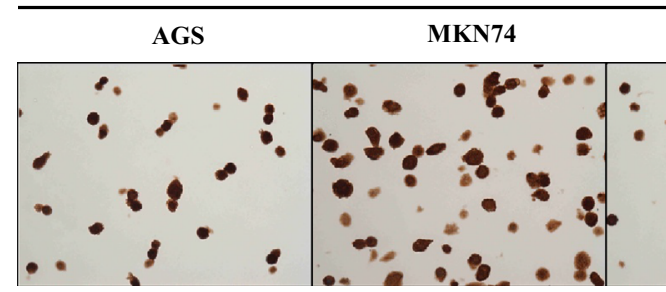

SNU719
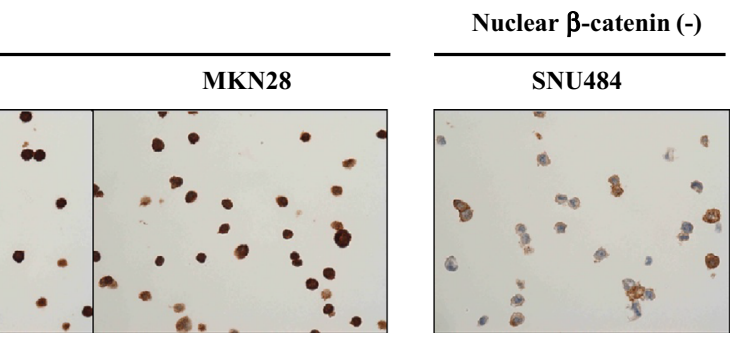

Fig. 5 Expression of LGR5 in the gastric cancer (GC) cell lines. a Fifteen GC cell lines were examined to determine the levels of endogenous LGR5, and five of them showed relatively higher LGR5 expression than the other ten GC cell lines. Asterisks indicate a group of LGR5-high cell lines. b GC cell lines with high LGR5 expression were demonstrated to have higher levels of ASCL2 than those with low LGR5 expression. c Immunocytochemistry showed that the AGS, MKN-74, SNU-719, and MKN-28 cell lines are positive for nuclear $\beta$-catenin, whereas the SNU-484 cell line is negative. Magnification $\times 400$
LGR5-positive cells, we could not conduct isolation and subsequent functional experiments, which are considered the gold standard, to assess the potential of stem cell markers. Instead, in this study, we revealed the expression of LGR5 in a large number of human GC cases by RNA ISH and its significance for the prognosis to explore the biological implications of LGR5 in GC patients.

LGR5 mRNA was shown to be overexpressed in many types of cancers, such as colon cancer [16, 28], hepatocellular carcinoma [29], esophageal cancer [30], and basal cell carcinoma [31], compared with corresponding normal tissues. In addition, we previously demonstrated overexpression of LGR5 in gastric adenomas compared with normal gastric mucosa [25]. Unexpectedly, however, we found that the mean level of LGR5 in GCs was significantly lower than that in matched noncancerous gastric tissues. This result may be explained by the fact that noncancerous gastric tissues obtained from the same stomach of GC patients are highly likely to harbor precancerous lesions, especially abundant foci of intestinal metaplasia. It is well known that intestinal-type GCs develop through a multistep process (chronic atrophic gastritis, intestinal metaplasia, and dysplasia), and most GCs are found in the background of intestinal metaplasia
[32]. Given the remarkable increase of LGR5 expression in intestinal metaplasia [25], variable degrees of intestinal metaplasia in noncancerous gastric mucosa could result in higher levels of LGR5 than in GCs. Although Yamanoi et al. [18] have reported upregulation of LGR5 in advanced GC tissues in comparison with noncancerous tissues, in which only 17 of 73 corresponding normal tissues were included for the comparison owing to the inadequate sample quality, which might have caused sampling bias. Also, the difference in the extent of intestinal metaplasia between study populations can lead to the discrepancy of the results in GCs.

By RNA ISH, we found that only $7 \%$ of GCs expressed LGR5 mRNA. Notably, LGR5 expression was observed more frequently in older age (older than 65 years). Age differences in LGR5 positivity may be related to the fact that LGR5 expression is higher in intestinal-type GCs (well to moderately differentiated GCs) than in diffuse type GCs (poorly differentiated and signet ring cell type GCs) since diffuse-type GCs are commoner in the young age group [33]. It has been shown that LGR5 expression in gastric adenomas is positively correlated with nuclear $\beta$-catenin expression [25]. Likewise, we found that LGR5 positivity was higher in GCs with nuclear $\beta$-catenin expression. This close 


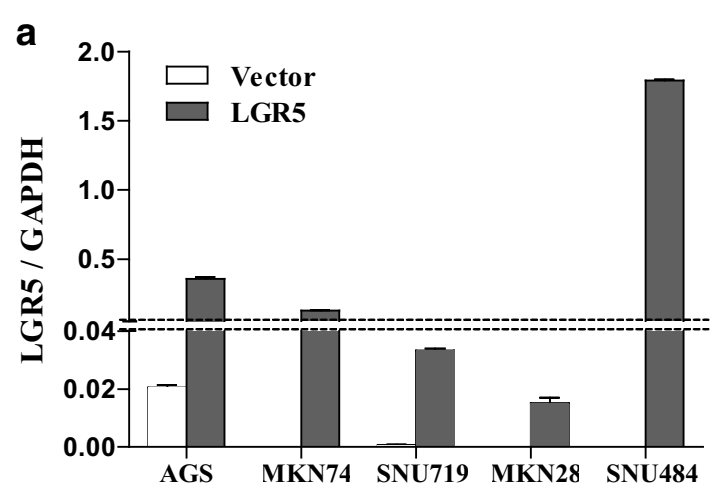

C
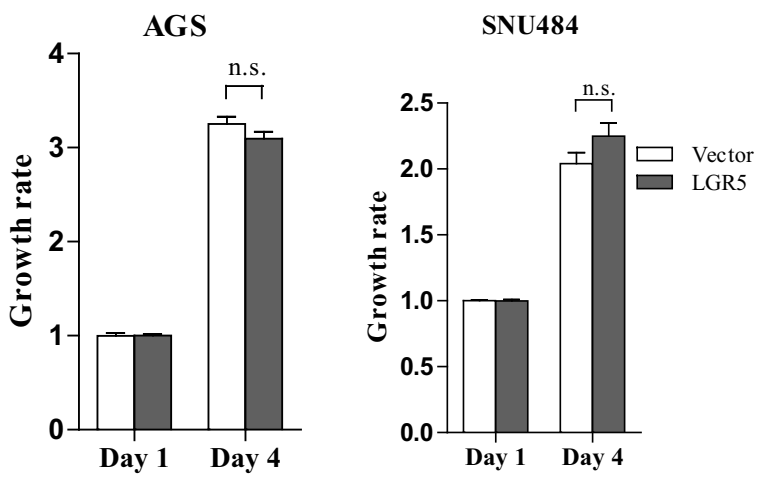

b

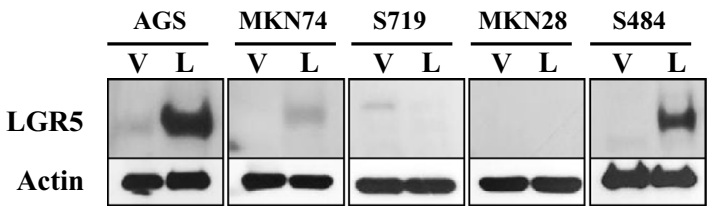

V: Vector, L: LGR5

d

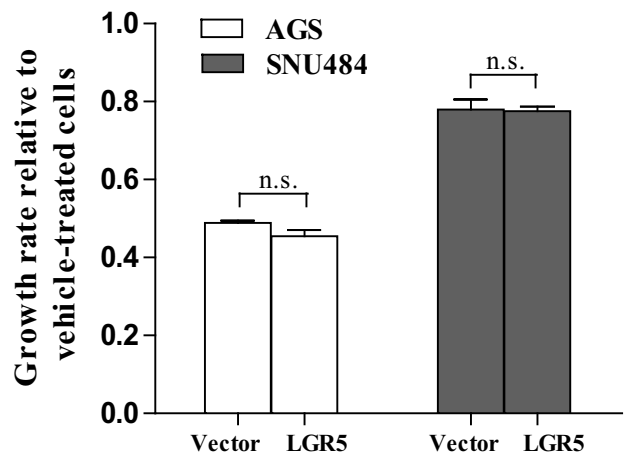

e

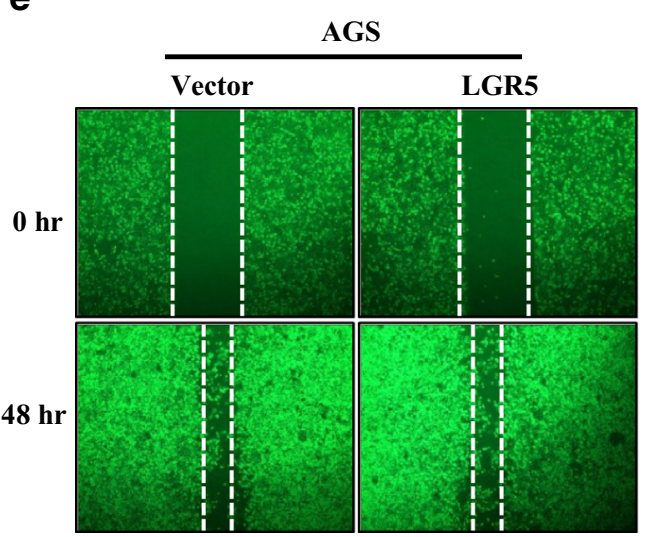

Fig. 6 Effects of LGR5 expression on the growth and chemosensitivity of gastric cancer cells. a Reverse transcription PCR analysis showed increased LGR5 transcripts in all gastric cancer cells transfected with LGR5. b Western blot analysis confirmed that only the AGS and SNU-484 cell lines expressed a high level of LGR5 protein. $\mathbf{c}$ There was no difference in the growth rate between LGR5-

correlation between LGR5 and increased Wnt signaling was also reported in colon cancers $[15,34]$ as well as hepatocellular carcinomas [29], in which overexpression of LGR5 was observed in $87.5 \%$ of hepatocellular carcinomas with mutation of $\beta$-catenin exon 3 . These findings are in accordance with the fact that LGR5 is one of the Wnt target genes and functions to augment canonical Wnt signals.

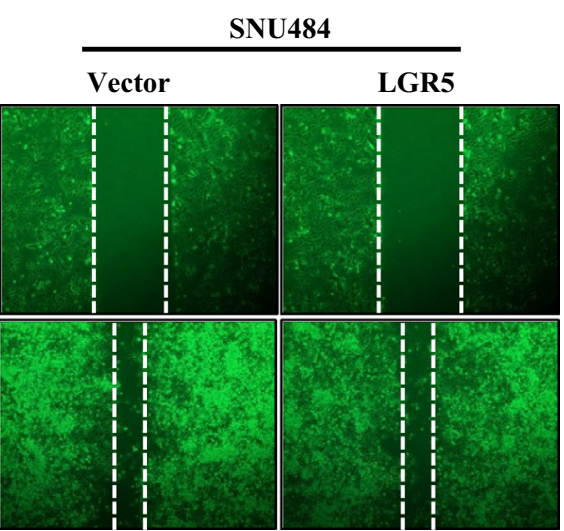

overexpressing and control cells. $\mathbf{d}$ No difference was observed in the relative cell growth $48 \mathrm{~h}$ after exposure to 5 -fluorouracil $(100 \mu \mathrm{g} / \mathrm{ml})$ between vector and LGR5-transfected cells. e Migration was evaluated by a wound healing assay, showing no difference in migration activity between control and LGR5 expression groups at $48 \mathrm{~h}$ after scratching

Most previous studies suggested that LGR5 expression is correlated with worse clinical outcomes. The prognostic impact of LGR5 has been most extensively studied in colorectal cancers, and recently two meta-analyses have concluded that LGR5 is related to the reduced overall survival in colorectal cancers [22,35]. In addition, Nakata et al. [21] reported that LGR5 expression increased with 
glioma progression and correlated with an adverse outcome, and Becker et al. [30] showed that high LGR5 expression was associated with worse survival in esophageal adenocarcinoma. For GCs, previous studies have reported inconsistent results. Xi et al. [24] showed that LGR5-positive patients had a significantly shorter survival time than LGR5-negative patients. On the other hand, Bauer et al. [36] suggested that LGR5 mRNA expression was not a prognostic marker in GCs even though LGR5 expression increased in tumors after neoadjuvant chemotherapy. Our data show that LGR5 expression is not an overall prognostic marker for GCs, but it is a poor prognostic marker when restricted to GCs with nuclear $\beta$ catenin expression. Given that LGR5 functions to enhance Wnt signaling, this finding suggests the possibility that LGR5 expression contributes to aggressive behavior of GCs with an activated Wnt pathway.

Although LGR5 expression in colon cancers is usually believed to be associated with poor survival, some studies have reported conflicting results regarding the prognostic influence of LGR5. Ziskin et al. [14] and Takahashi et al. [37] have shown no link between LGR5 expression and overall survival of colon cancer patients. These two studies examined LGR5 mRNA by RNA ISH or real time-PCR, whereas all the other studies reporting worse correlation of LGR5 with survival were based on the detection of LGR5 protein by immunohistochemistry. Thus, it seems that the difference in the method to detect LGR5 expression may be responsible for the discrepancy in the prognostic implications of LGR5. In fact, there is no reliable and commercially available antibody against LGR5 for use in immunohistochemistry, whereas it was clearly proved that LGR5-expressing cells on human FFPE samples can be specifically marked by RNA ISH [25]. Therefore, we believe that measuring mRNA either by RNA ISH or by RT-PCR would currently be more appropriate to evaluate the clinical significance of LGR5 with human specimens.

To determine whether LGR5 is functionally involved in the progression of GCs with aberrant Wnt activation, we performed in vitro growth and migration assays with GC cell lines with nuclear $\beta$-catenin expression. Unfortunately, however, we could not find any growth benefit with LGR5 expression in GC cells. One possible explanation for this result is the lack of an appropriate microenvironment in the in vitro experiments because Wnt signaling is also regulated by interactions between tumor cells and stromal cells. For instance, myofibroblasts expressing a high level of hepatocyte growth factor can activate Wnt signaling and even restore the stem cell phenotype in more differentiated tumor cells [38]. The Hedgehog and Notch pathways are also known to be deeply involved in the cancer stem cell properties [4]. Thus, our results suggest that LGR5 overexpression alone is not sufficient to recapitulate the molecular characteristics of LGR5-positive cells in GC. Maybe more complex and coordinated signaling pathways are required to fully activate the Wnt signaling and in turn affect the biological behavior of GC cells. We cannot rule out the possibility that LGR5 expression may be more of an indicator representing a stem cell phenotype than a functional factor in maintaining the stem cell properties. If so, induced expression of LGR5 in GCs is not an appropriate way to evaluate the significance of LGR5 as a prognostic marker. As mentioned earlier, isolation of LGR5-expressing cells from GC specimens and their subsequent molecular characterization of is required to help unravel the significance of LGR5 as a prognostic marker in GCs.

In summary, LGR5 expression of GCs was lower than that of the corresponding noncancerous gastric tissues. Only $7 \%$ of GCs examined were positive for LGR5, which was correlated with older age, well-differentiated histologic type, and nuclear $\beta$-catenin expression. Although LGR5 was not a prognostic marker for all GC cases, it was associated with worse survival for GCs with nuclear $\beta$ catenin expression, suggesting a functional role of LGR5 in cancer progression of GCs with abnormally enhanced Wnt signaling. However, in vitro assays provided no evidence of a survival benefit of LGR5, demanding further studies on the biological roles of LGR5 to facilitate the investigation of its potential as a candidate marker for the targeted therapy of GCs.

Acknowledgment This research was supported by a grant from the Korea Health Technology R\&D Project through the Korea Health Industry Development Institute, funded by the Ministry of Health and Welfare, Republic of Korea (grant number HI14C1277).

\section{References}

1. Ferlay J, Soerjomataram I, Dikshit R, Eser S, Mathers C, Rebelo M, Parkin DM, Forman D, Bray F. Cancer incidence and mortality worldwide: sources, methods and major patterns in GLOBOCAN 2012. Int J Cancer. 2014;136(5):E359-86.

2. Yang W, Raufi A, Klempner SJ. Targeted therapy for gastric cancer: molecular pathways and ongoing investigations. Biochim Biophys Acta. 2014;1846(1):232-7.

3. Bang Y-J, Van Cutsem E, Feyereislova A, Chung HC, Shen L, Sawaki A, Lordick F, Ohtsu A, Omuro Y, Satoh T. Trastuzumab in combination with chemotherapy versus chemotherapy alone for treatment of HER2-positive advanced gastric or gastro-oesophageal junction cancer (ToGA): a phase 3, open-label, randomised controlled trial. Lancet. 2010;376(9742):687-97.

4. Stojnev S, Krstic M, Ristic-Petrovic A, Stefanovic V, Hattori T. Gastric cancer stem cells: therapeutic targets. Gastric Cancer. 2014;17(1):13-25.

5. Barker N, van Es JH, Kuipers J, Kujala P, van den Born M, Cozijnsen M, Haegebarth A, Korving J, Begthel H, Peters PJ. Identification of stem cells in small intestine and colon by marker gene Lgr5. Nature. 2007;449(7165):1003-7.

6. Barker N, Huch M, Kujala P, van de Wetering M, Snippert HJ, van Es JH, Sato T, Stange DE, Begthel $\mathrm{H}$, van den Born M. 
$\mathrm{Lgr}^{+\mathrm{ve}}$ stem cells drive self-renewal in the stomach and build long-lived gastric units in vitro. Cell Stem Cell. 2010;6(1):25-36.

7. Jaks V, Barker N, Kasper M, Van Es JH, Snippert HJ, Clevers H, Toftgård R. Lgr5 marks cycling, yet long-lived, hair follicle stem cells. Nat Genet. 2008;40(11):1291-9.

8. Barker N, Rookmaaker MB, Kujala P, Ng A, Leushacke M, Snippert H, van de Wetering M, Tan S, Van Es JH, Huch M. $\mathrm{Lgr5}^{+\mathrm{ve}}$ stem/progenitor cells contribute to nephron formation during kidney development. Cell Rep. 2012;2(3):540-52.

9. Plaks V, Brenot A, Lawson DA, Linnemann JR, Van Kappel EC, Wong KC, de Sauvage F, Klein OD, Werb Z. Lgr5-expressing cells are sufficient and necessary for postnatal mammary gland organogenesis. Cell Rep. 2013;3(1):70-8.

10. Flesken-Nikitin A, Hwang C-I, Cheng C-Y, Michurina TV, Enikolopov G, Nikitin AY. Ovarian surface epithelium at the junction area contains a cancer-prone stem cell niche. Nature. 2013;495(7440):241-5.

11. Huch M, Dorrell C, Boj SF, van Es JH, Li VS, van de Wetering M, Sato T, Hamer K, Sasaki N, Finegold MJ. In vitro expansion of single Lgr5+ liver stem cells induced by Wnt-driven regeneration. Nature. 2013;494(7436):247-50.

12. Schepers AG, Snippert HJ, Stange DE, van den Born M, van Es $\mathrm{JH}$, van de Wetering $\mathrm{M}$, Clevers $\mathrm{H}$. Lineage tracing reveals Lgr5+ stem cell activity in mouse intestinal adenomas. Science. 2012;337(6095):730-5.

13. Kemper K, Prasetyanti PR, De Lau W, Rodermond H, Clevers H, Medema JP. Monoclonal antibodies against Lgr5 identify human colorectal cancer stem cells. Stem Cells. 2012;30(11):2378-86.

14. Ziskin JL, Dunlap D, Yaylaoglu M, Fodor IK, Forrest WF, Patel R, Ge N, Hutchins GG, Pine JK, Quirke P, Koeppen H, Jubb AM. In situ validation of an intestinal stem cell signature in colorectal cancer. Gut. 2013;62(7):1012-23.

15. He S, Zhou H, Zhu X, Hu S, Fei M, Wan D, Gu W, Yang X, Shi D, Zhou J. Expression of Lgr5, a marker of intestinal stem cells, in colorectal cancer and its clinicopathological significance. Biomed Pharmacother. 2014;68(5):507-13.

16. Uchida H, Yamazaki K, Fukuma M, Yamada T, Hayashida T, Hasegawa H, Kitajima M, Kitagawa Y, Sakamoto M. Overexpression of leucine-rich repeat-containing $\mathrm{G}$ protein-coupled receptor 5 in colorectal cancer. Cancer Sci. 2010;101(7):1731-7.

17. Simon E, Petke D, Böger C, Behrens H-M, Warneke V, Ebert M, Röcken C. The spatial distribution of LGR5+ cells correlates with gastric cancer progression. PLoS One. 2012;7(4):e35486.

18. Yamanoi K, Fukuma M, Uchida H, Kushima R, Yamazaki K, Katai H, Kanai Y, Sakamoto M. Overexpression of leucine-rich repeat-containing $\mathrm{G}$ protein-coupled receptor 5 in gastric cancer. Pathol Int. 2013;63(1):13-9.

19. Fukuma M, Tanese K, Effendi K, Yamazaki K, Masugi Y, Suda $\mathrm{M}$, Sakamoto M. Leucine-rich repeat-containing G protein-coupled receptor 5 regulates epithelial cell phenotype and survival of hepatocellular carcinoma cells. Exp Cell Res. 2013; 319(3):113-21.

20. Ryuge S, Sato Y, Jiang S-X, Wang G, Kobayashi M, Nagashio R, Katono K, Iyoda A, Satoh Y, Masuda N. The clinicopathological significance of Lgr5 expression in lung adenocarcinoma. Lung Cancer. 2013;82(1):143-8.

21. Nakata S, Campos B, Bageritz J, Lorenzo Bermejo J, Becker N, Engel F, Acker T, Momma S, Herold-Mende C, Lichter P. LGR5 is a marker of poor prognosis in glioblastoma and is required for survival of brain cancer stem-like cells. Brain Pathol. 2013;23(1):60-72.

22. Han Y, Xue X, Jiang M, Guo X, Li P, Liu F, Yuan B, Shen Y, Guo X, Zhi Q. LGR5, a relevant marker of cancer stem cells, indicates a poor prognosis in colorectal cancer patients: a metaanalysis. Clin Res Hepatol Gastroenterol. 2014;39(2):267-73.
23. Xi H, Cai A, Wu X, Cui J, Shen W, Bian S, Wang N, Li J, Lu C, Song Z. Leucine-rich repeat-containing G-protein-coupled receptor 5 is associated with invasion, metastasis, and could be a potential therapeutic target in human gastric cancer. Br J Cancer. 2014;110(8):2011-20.

24. Xi H-Q, Cui J-X, Shen W-S, Wu X-S, Bian SB, Li J-Y, Song Z, Wei B, Chen L. Increased expression of Lgr5 is associated with chemotherapy resistance in human gastric cancer. Oncol Rep. 2014;32(1):181-8.

25. Jang BG, Lee BL, Kim WH. Distribution of LGR5+ cells and associated implications during the early stage of gastric tumorigenesis. PLoS One. 2013;8(12):e82390.

26. de Lau W, Barker N, Low TY, Koo B-K, Li VS, Teunissen H, Kujala P, Haegebarth A, Peters PJ, van de Wetering M. Lgr5 homologues associate with Wnt receptors and mediate R-spondin signalling. Nature. 2011;476(7360):293-7.

27. Glinka A, Dolde C, Kirsch N, Huang YL, Kazanskaya O, Ingelfinger $\mathrm{D}$, Boutros $\mathrm{M}$, Cruciat $\mathrm{CM}$, Niehrs C. LGR4 and LGR5 are R-spondin receptors mediating Wnt/ $\beta$-catenin and Wnt/PCP signalling. EMBO Rep. 2011;12(10):1055-61.

28. Merlos-Suárez A, Barriga FM, Jung P, Iglesias M, Céspedes MV, Rossell D, Sevillano M, Hernando-Momblona X, da Silva-Diz V, Muñoz P. The intestinal stem cell signature identifies colorectal cancer stem cells and predicts disease relapse. Cell Stem Cell. 2011;8(5):511-24.

29. Yamamoto Y, Sakamoto M, Fujii G, Tsuiji H, Kenetaka K, Asaka M, Hirohashi S. Overexpression of orphan G-protein-coupled receptor, Gpr49, in human hepatocellular carcinomas with $\beta$ catenin mutations. Hepatology. 2003;37(3):528-33.

30. Becker L, Huang Q, Mashimo H. Lgr5, an intestinal stem cell marker, is abnormally expressed in Barrett's esophagus and esophageal adenocarcinoma. Dis Esophagus. 2010;23(2):168-74.

31. Tanese K, Fukuma M, Yamada T, Mori T, Yoshikawa T, Watanabe W, Ishiko A, Amagai M, Nishikawa T, Sakamoto M. G-protein-coupled receptor GPR49 is up-regulated in basal cell carcinoma and promotes cell proliferation and tumor formation. Am J Pathol. 2008;173(3):835-43.

32. Gonzalez CA, Sanz-Anquela JM, Gisbert JP, Correa P. Utility of subtyping intestinal metaplasia as marker of gastric cancer risk. A review of the evidence. Int J Cancer. 2013;133((5):):1023-32.

33. Jeong O, Park Y-K. Clinicopathological features and surgical treatment of gastric cancer in South Korea: the results of 2009 nationwide survey on surgically treated gastric cancer patients. J Gastric Cancer. 2011;11(2):69-77.

34. Fan X-S, Wu H-Y, Yu H-P, Zhou Q, Zhang Y-F, Huang Q. Expression of Lgr5 in human colorectal carcinogenesis and its potential correlation with $\beta$-catenin. Int $J$ Colorectal Dis. 2010;25(5):583-90.

35. Chen Q, Zhang X, Li W-M, Ji Y-Q, Cao H-Z, Zheng P. Prognostic value of LGR5 in colorectal cancer: a meta-analysis. PLoS One. 2014;9(9):e107013.

36. Bauer L, Langer R, Becker K, Hapfelmeier A, Ott K, Novotny A, Höfler H, Keller G. Expression profiling of stem cell-related genes in neoadjuvant-treated gastric cancer: a NOTCH2, GSK3B and $\beta$-catenin gene signature predicts survival. PLoS One. 2012;7(9):e44566.

37. Takahashi H, Ishii H, Nishida N, Takemasa I, Mizushima T, Ikeda M, Yokobori T, Mimori K, Yamamoto H, Sekimoto M. Significance of $\mathrm{Lgr5}^{+\mathrm{ve}}$ cancer stem cells in the colon and rectum. Ann Surg Oncol. 2011;18(4):1166-74.

38. Vermeulen L, Felipe De Sousa EM, van der Heijden M, Cameron K, de Jong JH, Borovski T, Tuynman JB, Todaro M, Merz C, Rodermond $\mathrm{H}$. Wnt activity defines colon cancer stem cells and is regulated by the microenvironment. Nat Cell Biol. 2010;12(5):468-76. 